\title{
Parallel Methods in Time Dependent Approaches to Reactive Scattering Calculations
}

\author{
Valentina Piermarini ${ }^{1}$, Leonardo Pacifici ${ }^{1}$, Stefano Crocchianti ${ }^{1}$, \\ Antonio Laganà ${ }^{1}$, Giuseppina D'Agosto ${ }^{2}$, and Sergio Tasso ${ }^{2}$ \\ 1 Dipartimento di Chimica, Università di Perugia, Via Elce di Sotto, 8, \\ 06123 Perugia, Italy \\ 2 Centro Ateneo Servizi Informatici, Università di Perugia, \\ 06123 Perugia, Italy
}

\begin{abstract}
The possibility of implementing a suitable model to parallelize time-dependent approaches to the calculation of quantum reactive probabilities of elementary atom diatom processes based on collocation methods is investigated. Problems arising when adopting a coarse grain model by distributing fixed total angular momentum quantum number $J$ calculations are discussed. A comparison is made with finer grain models based either on a domain decomposition due to the distribution of the various projections $\Lambda$ of $J$ or to a partitioning of the collocation matrices among the available processors.

Measurements performed indicate that a finer grain parallelism is a proper solution if the communications can be confined into reasonable limits. Otherwhise, fine grain parallelism is only an "extrema ratio" for dealing with problems based on matrix representations too large to be dealt by a single processor.
\end{abstract}

\section{Introduction}

Parallelization of computer codes devoted to the calculation of the properties of reacting chemical systems has received increasing attention in recent years 1 . This is motivated by the need for building realistic molecular simulations of several processes of interest for environmental monitoring and control, technology and material research and development, drug and enzyme design.

Up to now this has been the realm of molecular mechanics and classical dynamics that is of approaches based upon the assumption that atoms behave as mass points or microscopic solids moving on a potential energy surface according to the laws of classical mechanics. More recently, especially for a few atom systems, progress has been made in building computational tools based on the more rigorous quantum mechanical assumption that atoms and molecules need to be represented by wavefunctions.

Quantum mechanical calculations, however, are more difficult to carry out than classical ones. They, in fact, require large memories to store the information about the basis set (and related integrals, eigenvalues, eigenvectors, etc.) in which the wavefunction of the molecular system being considered has been expanded. 
This technique is largely preferred in time independent quantum approaches. As an alternative, the wavefunction of the molecular system can be represented as values on a grid (collocation methods). This technique is preferred for time dependent quantum approaches. In this case, larger memories are needed to store the values of the wavefunction at the chosen grid points.

In collaboration with other laboratories, we have developed computational procedures able to describe reactions and calculate related observables. These procedures are highly time consuming and have been analysed for parallelization. Extensive work has been carried out to parallelize computer codes based on time independent quantum approaches. In particular, both task farm and pipe-line models have been considered for the implementation of full 22 and reduced dimensionality 3 computational procedures on parallel architectures.

On the contrary, only limited work has been performed for parallelizing time dependent approaches. Aim of this paper is to describe the implementation of some parallel models for computer codes based on time dependent methods. In section 2 a brief description of the time dependent approach is given. In section 3 a coarse grain parallelization scheme is discussed. In section 4 progress towards a fine grain parallelization scheme is illustrated.

\section{The Quantum Time Dependent Computational Procedure}

The case considered in this paper is the atom-diatom reaction:

$$
\mathrm{A}+\mathrm{BC}(v, j) \longrightarrow \mathrm{AB}\left(v^{\prime}, j^{\prime}\right)+\mathrm{C}
$$

where the reactant diatomic molecule $\mathrm{BC}$ is in its vibrotational $(v j)$ state and the product diatomic molecule $\mathrm{AB}$ or $\mathrm{AC}$ is in its vibrotational $\left(v^{\prime} j^{\prime}\right)$ state (we use primed quantities for products, unprimed for reactants). The time-dependent method used in this work makes use of a grid representation of the wavepacket for distance coordinates and a basis set expansion for the angular coordinate. The propagation in time of the wave packet considers only its real part [45].

At the very beginning $(t=0)$, for a given value of the total angular momentum quantum number $J$ and its projection $\Lambda$ on the $\mathrm{z}$ axis of a body fixed frame, the system wavefunction $\Psi^{J \Lambda}$ is defined as

$$
\begin{gathered}
\Psi^{J \Lambda}(R, r, \Theta ; t)=\left(\frac{8 \alpha}{\pi}\right)^{\frac{1}{4}} e^{-\alpha\left(R-R_{0}\right)^{2}} \\
\cdot e^{-i k\left(R-R_{0}\right)} \cdot \varphi_{v j}^{B C}(r) \cdot P_{j}^{\Lambda}(\Theta) .
\end{gathered}
$$

where $R, r$ and $\Theta$ are the Jacobi internal coordinates of the reactant atom diatom system. In eq. 2] $e^{-i k\left(R-R_{0}\right)}$ is a phase factor which gives the initial wave packet a relative kinetic energy towards the interaction region, $\varphi_{v j}^{B C}(r)$ is the initial diatomic molecule BC wavefunction (for the vibrational state $v$ and the rotational state $j$ ) expressed in the Jacobi coordinates of the reactant arrangement, $P_{j}^{\Lambda}(\Theta)$ is the normalised associated Legendre polynomial and $k$ 
is the wavevector which determines the relative kinetic energy of the collisional partners [4]. In this way, the wavefunction is defined for a given accessible state of the reactants and a given collisional energy range.

To move the wavepacket out of the reactant region, the integration in time of the time-dependent Schrödinger equation

$$
i \hbar \frac{\partial}{\partial t} \Psi^{J \Lambda}(R, r, \Theta ; t)=\hat{H} \Psi^{J \Lambda}(R, r, \Theta ; t)
$$

is performed. The hamiltonian $\hat{H}$ consists of a kinetic part $(\hat{T})$ and a potential part $(V)$ which are multiplicative in the momentum and in the coordinate space, respectively. To exploit this property the application of $\hat{H}$ on $\Psi$ is performed by switching from the coordinate to the momentum space and viceversa for each coordinate. This is the most demanding part of the code in terms of computing time. In fact, this requires that a back and forth Fourier trasform of the wavefunction for all the coordinates involved is repeated at each time step. As an alternative, one can apply a discrete variable (DVR) method for which there is no need to perform the fast Fourier transform although interaction of neighbouring elements of the wave function need to be considered.

In order to analyse the dynamics of the reactive process we require the wavefunction to be expanded in terms of the final diatomic molecule $\mathrm{AB}$ wavefunction $\left(\varphi_{v^{\prime} j^{\prime}}^{A B}\left(r^{\prime}\right)\right.$ where $r^{\prime}, R^{\prime}$ and $\Theta^{\prime}$ are the Jacobi coordinates of the product arrangement). From the cut of the wavepacket at the analysis line located far away in the asymptotic region, one can evaluate the time dependent coefficients $C_{v j \Lambda, v^{\prime} j^{\prime} \Lambda^{\prime}}^{J}(t)$ of the expansion. By half Fourier transforming the time-dependent coefficients $C_{v j \Lambda, v^{\prime} j^{\prime} \Lambda^{\prime}}^{J}(t)$ one obtains a set of energy-dependent coefficients whose square modulus is the reaction probability.

The computer code performing the propagation in time of the wavepacket is TIDEP. It carries out iteratively the propagation of the real part of the wavefunction and stores at each time step the value of the $C_{v j \Lambda, v^{\prime} j^{\prime} \Lambda^{\prime}}^{J}(t)$ coefficients. The analysis of the coefficients to work out reaction probabilities is performed off line by another program called TIDAN.

\section{The Coarse Grain Parallelization}

Parallelization efforts were concentrated on TIDEP since this program is the most time consuming component of the computational procedure (the propagation step has to be iterated for about $10^{4} \div 10^{5}$ times). The structure of TIDEP is:

Read input data: $v, j, k$, masses, $\ldots$

Perform preliminary calculations

LOOP on $J$

LOOP on $t$

LOOP on $\Lambda$ 
Perform time step integration

Perform the asymptotic analysis

Store $C(t)$ coefficients

END loop on $\Lambda$

END loop on $t$

END loop on $J$

Calculate final quantities

Print outputs

As can be seen from the scheme given above, calculations are performed at a given range of energy, at a fixed value of the vibrotational quantum number $(v j)$ of the reactant diatom and a single value of the total angular momentum quantum number $J$. Therefore, the coarsest grain of parallelism that can be adopted is the one distributing the calculation for a given vibrotational state, a given interval of translational energy and a fixed value of $J$. In this case, the characteristics of the various tasks are so different that a task farm dynamically assigning the computational workload can be adopted. This very coarse grain approach was fruitfully implemented on a cluster of powerful workstations.

To carry out the calculations the value of the physical parameters was chosen to be that of the $\mathrm{O}\left({ }^{1} \mathrm{D}\right)+\mathrm{HCl}$ atom diatom reaction [5]. Accordingly, the mass values were chosen to be $15.9949 \mathrm{amu}$ for $\mathrm{O}, 1.00783 \mathrm{amu}$ for $\mathrm{H}$ atom and $34.96885 \mathrm{amu}$ for $\mathrm{Cl}$. The energy range covered by the calculation was approximately $1 \mathrm{eV}$, the initial vibrotational state used for the test was $v=0$ and $j=$ 0 . The potential energy surface used for the calculations is described in ref. $[5$ where other details are also given. Two types of gridsize were used for $R^{\prime}$ and $r^{\prime}$ (the angular part was in both cases expanded over 80 basis functions): (a) 127 $\times 119$ points; (b) $251 \times 143$ points. Time propagation iterates for about 40000 steps to properly diffuse the wavepacket at $J=0$. Production runs take about 3 weeks on a Sylicon Graphics PowerChallenge supercomputer. This means also that to calculate a state to state cross section or, even worse, a state to state rate coefficient, the amount of time needed to perform the calculation goes beyond any acceptable limit if simplifications are not introduced. In fact, to evaluate a vibrational state selected rate coefficient, the calculation needs to be for all the reactant rotational states $j$ populated at the temperature considered. In addition, the calculations need to be converged with $J$ and convergence is usually reached only at $J>100$. This increases enormously the computational load not only because calculations have to be repeated for all $J$ values but also because the dimension of the matrices to be handled in a single $J$ calculation is $J+1$ times larger than that of $J=0$. As a matter of fact, the computing time, that depends on the third power of the matrix dimension, rapidly becomes exceedingly large even at small $J$ values. This makes the calculation unfeasible on the machines presently available for academic use at the large scale computing facilities in Europe.

For the same reason this parallel model is even less applicable to four or more atom reactions. 


\section{The Finer Coarse Grain Parallelization}

A next lower level of parallelization is the one based on the combined distribution of fixed $J$ and fixed $\Lambda$ calculations. As it has been already shown above, there is no problem in distributing fixed $J$ calculations: $J$ is a good quantum number (i.e. calculations for different $J$ values are fully decoupled) and, accordingly, the parallelization on $J$ is natural. On the contrary, the decoupling of $\Lambda$ is not natural since one has to introduce physical constraints of the centrifugal sudden type (i.e. the projection of $J$ on the $z$ axis of the body fixed frame remains constant during the collision). This allows to perform separately the step-propagation of the wavepacket for blocks of fixed $\Lambda$ values and the recombination of the various contributions only at the end of the propagation step. This is a key feature of the adopted computational scheme since it allows a decomposition of the domain of the wavepacket that otherwhise would lead to a drastic increase of the demand for memory when $J$ increases.

Such a parallel model was first tested [6] on the Cray T3E of EPCC (Edinburgh, UK) for the simplest case of $J=0$ and $J=1$. In this case, only three pairs of $J$ and $\Lambda$ values needed to be considered and only three nodes were used (this is the smallest non zero $J$ calculation allowing an investigation of the model proposed). To further save on computing time the integration process was truncated after a few steps (computing time depends linearly on the number of propagation steps and their reduction leads only to a slight underestimate of the speedup). Measured speedups are 2.6 and 2.5 for propagation grids (a) and (b), respectively. This clearly indicates that the proposed model is quite effective in reducing computing times. However, for this parallel model $\mathrm{I} / \mathrm{O}$ is a real bottleneck, since as reported in ref. [6, it accounts for about $1 / 5$ of the overall computing time. Moreover, I/O times of node zero (through which all I/O is channeled) are four order of magnitude larger than those of the workers. This clearly indicates that there is still room for improvement. In fact, by entirely conveying I/O through the master node, one has the advantage of simplifying $\mathrm{I} / \mathrm{O}$ operations at the expenses of overloading the master node.

Accordingly, when generalizing the model to higher $J$ values node zero was exclusively dedicated to acting as a master and the centralized management of I/O was abandoned. On the contrary, the feature of carrying out fixed $J$ calculations in pairs (including all the $J+1$ component of $\Lambda$ ) was kept. The fact that the parallelization is performed on $J$ sets some limits to the maximum value of the total angular momentum quantum number that can be handled by the program. Using this model, in fact, the maximum value of $J$ has to be 3 units lower than the number of processors. In addition, in order to keep all the processors busy, the pairs of $J$ values simultaneously running have to sum up to the maximum allowed value. As an example, for a 16 node machine the maximum allowed value of $J$ is 13 . In this case, in fact, one node acts as a master, at least one node is reserved to the smaller $J$ calculation and the remaining ones to the larger $J$ calculation. Their values are chosen so as to make the sum equal to 13 (the number of $\Lambda$ values is $J+1$ since it varies from 0 to $J$ ). The looping on $J$ starts from the pair $J=0$ (one $\Lambda$ value) and $J=13$ (fourteen $\Lambda$ values) and 
then it continues by rising the lower $J$ value and lowering the higher one until the ascending and descending sequences converge.

To evaluate the performances of the model, the calculations were performed on the Origin 3800 of Cineca (Bologna, I) using the same set of parameters adopted for the tests described above (grid (a)) and reducing the basis set expansion for the angular part to 10. Measured times are shown in Table 1 where the average node computing time (in second) is given for different $J$ values.

Table 1. Execution time in seconds

\begin{tabular}{lllllllllllllll}
\hline $\mathrm{J}$ & 0 & 1 & 2 & 3 & 4 & 5 & 6 & 7 & 8 & 9 & 10 & 11 & 12 & 13 \\
\hline time/s & 4400 & 4440 & 4480 & 4540 & 4680 & 4680 & 4760 & 5080 & 5160 & 5120 & 5240 & 5280 & 5360 & 5400 \\
\hline
\end{tabular}

As clearly shown by the results reported in the Table, the computing time per node (averaged over the various values of $\Lambda$ ) increases with $J$. This indicates that the increase of communication time associated with an increase in the number of allowed $\Lambda$ values penalizes the efficiency of the code. However, its entity is small and the model shows to be quite effective in reducing the overall computing time.

\section{Fine Grain Parallelization}

Further attempts have been made to evaluate the possibility of pushing the parallelization to a very fine granularity. This turns out to be useful either when a specific $J$ value calculation needs to be carried out or when the dimensionality of the problem becomes so large (like in the case of polyatomic reactions) that it does not fit into the individual node memory. This particularly applies when computer center policies set severe limitations on memory and on the amount of computing time assigned to individual jobs.

To work out a finer grain parallel model the fixed angle version of the code making use of the DVR technique to perform the propagation was used. Related operations are performed inside the routine $a v$. Inside $a v$ two matrix times vector and one vector times vector operations are performed according to the following computational scheme:

LOOP of $i v$ from 1 to $n v$

LOOP of $i r$ from 1 to $n r$

$\mathrm{a}($ ir,iv $)=0$

END loop of $i r$

END loop of $i v$

LOOP of $i v$ from 1 to $n v$

LOOP of $i$ from 1 to $n r$ 
LOOP of $i p$ from 1 to $n r$

$$
\mathrm{a}(\mathrm{i}, \mathrm{iv})=\mathrm{a}(\mathrm{i}, \mathrm{iv})+\mathrm{b}(\mathrm{i}, \mathrm{ip})^{*} \mathrm{c}(\mathrm{ip}, \mathrm{iv})
$$

END loop of $i p$

END loop of $i$

END loop of $i v$

LOOP of $i$ from 1 to $n r$

LOOP of $i v$ from 1 to $n v$

LOOP of $i v p$ from 1 to $n v$

$$
\mathrm{a}(\mathrm{i}, \mathrm{iv})=\mathrm{a}(\mathrm{i}, \mathrm{iv})+\mathrm{s}(\mathrm{iv}, \mathrm{ivp}) * \mathrm{c}(\mathrm{i}, \mathrm{ivp})
$$

END loop of $i v p$

END loop of $i v$

END loop of $i$

LOOP of $i v$ from 1 to $n v$

LOOP of $i$ from 1 to $n r$

$$
\mathrm{a}(\mathrm{i}, \mathrm{iv})=\mathrm{a}(\mathrm{i}, \mathrm{iv})+\mathrm{v}(\mathrm{i}, \mathrm{iv})^{*} \mathrm{c}(\mathrm{i}, \mathrm{iv})
$$

END loop of $i$

END loop of $i v$

When all the matrices involved are distributed per (groups of) columns among a certain number of nodes, all the operations sketched above imply a quite significant amount of communication to allow the nodes have the updated version of the matrices involved. Test runs were performed on the IBM SP2 of the computer Center of the University of Perugia. For the measurements use was made of 5 processors of which one acts as a master and runs the main program, transfers the information from and to the nodes, combines the pieces of information arriving from the nodes in a form suitable for redistribution and further manipulation. The ratio s/p between measured sequential (single node) and parallel (five nodes) execution times is given in Table 2 for different values

\begin{tabular}{|c|c|}
\hline Dimension 80 & 400500600 \\
\hline $\mathrm{s} / \mathrm{p} \quad 9$. & $\begin{array}{llll}5 & 1.2 & 1.0 & 0.98\end{array}$ \\
\hline
\end{tabular}
of the collocation matrix dimension. The Table clearly shows that the parallel

Table 2. Ratio of sequential/parallel execution time

code is outperformed by the sequential one for small dimensions of the matrices. Break even occurs at dimension 500 for which the two versions of the code take the same amount of time. For larger dimensions the parallel code outperforms the sequential one. This means that, according to measurements performed here, there is no advantage in parallelizing the code at this fine level of granularity unless further investigations and deep restructuring or, possibly, the use of clever 
parallelization tools [7] allow a significant reduction in communication time. The only convenience can be found in the fact that a fine grain parallelism allows to deal with matrices too large to be dealt by a single processor memory.

\section{Conclusions}

The investigation of suitable models for the parallelization quantum time dependent approaches to the calculation of reactive probabilities of elementary atom diatom processes has been carried out for approaches based on collocation methods. The various models investigated show that in order to make the computing time manageable the parallelization has to be pushed to a fairly low level. This has been exploited for time dependent approaches to chemical reactivity by distributing the propagation on time of the fixed angular momentum and fixed angular momentum projection wavepacket and regaining the coupling after each integration step. The model was found to be quite efficient and to break the calculation into a computational grain small enough to make the program run on a reasonable amount of time. On the contrary, when the parallelization is pushed to a lower level and the computational grain is made finer, the overhead due to communications has shown to be so heavy to make the efficiency of the parallel computational procedure very poor. The choice of such a fine grain parallelism is only justified by the need of tackling a problem having a matrix representation too large to be dealt by a single processor.

\section{References}

1. Laganà, A. : Innovative computing and detailed properties of elementary reactions using time independent approaches. Comp. Phys. Comm. 116 (1999) 1-16; Laganà, A., Crocchianti, S., Bolloni, A., Piermarini, V., Baraglia, R., Ferrini, R., Laforenza, D. : Computational granularity and parallel models to scale up reactive scattering calculations. Comp. Phys. Comm. 128 (2000) 295-314

2. Laganà, A., Crocchianti, S., Ochoa de Aspuru, G., Gargano, R., Parker, G.A.: Parallel time independent quantum calculations of atom diatom reactivity. Lecture Notes in Computer Science Vol. 1041. Springer-Verlag, Berlin Heidelberg New York (1995) 361-370; (1995) 361-370; Bolloni, A., Riganelli, A., Crocchianti, S., Laganà, A.: Parallel quantum scattering calculations applied to the dynamics of elementary reactions. Lecture Notes in Computer Science Vol. 1497. Springer-Verlag, Berlin Heidelberg New York (1998) 331-339

3. Baraglia, R., Laforenza, D., Laganà, A.: Parallelization strategies for a reduced dimensionality calculation of quantum reactive scattering cross sections on a hypercube machine. Lecture Notes in Computer Science Vol. 919. Springer-Verlag, Berlin Heidelberg New York (1995) 554-561

4. Balint-Kurti, G. G.: Time dependent quantum approaches to chemical reactivity. Lecture Notes in Chemistry Vol. 75. Springer-Verlag, Berlin Heidelberg New York (2000) $74-87$ 
5. Balint-Kurti, G.G., Dixon, R. N., Marston. C. C.: Grid methods for solving the Shrödinger equation and time dependent quantum dynamics of molecular photofragmentation and reactive scattering processes. International Reviews in Physical Chemistry 111 (1992) 317-344; V. Piermarini, V., Balint-Kurti, G.G., Gray, S., Gogtas, F., Hernandez, M.L., Laganà, A.: Wavepacket calculation of cross sections, product state distributions and branching ratios for the $\mathrm{O}\left({ }^{1} \mathrm{D}\right)+$ $\mathrm{HCl}$ reaction. J. Phys. Chem (in the press)

6. Piermarini, V., Laganà, A., Smith, L., Balint-Kurti, G. G., Allan, R. J.: Parallelism and granularity in time dependent approaches to reactive scattering calculations. PDPTA 5 (2000) 2879-2884

7. Vanneschi, M.: Heterogeneous High Performance Computing environment. Lecture Notes in Computer Science Vol. 1470.Springer-Verlag, Berlin Heidelberg New York (1998) 21-34 